\title{
Face Detection and Recognition using Open CV Based on Fisher Faces Algorithm
}

\author{
J. Manikandan, S. Lakshmi Prathyusha, P. Sai Kumar, Y. Jaya Chandra, M. Umaditya Hanuman
}

\begin{abstract}
Facial Recognition represents the event of a system which may determine the person with the assistance of a face using Computer Vision (Open CV). Face recognition is employed within the fields of Identity Recognition, police investigation and enforcement. It's a method of characteristic someone supported facial expression. This method is enforced in 2 stages. They're the training stage and therefore the testing stage. This study primarily consists of 3 elements, specifically face detection from the image, feature extraction and storing many reminder images, and recognition. Face finding rule is employed to detect the face from the given image. The foremost helpful and distinctive options of the face image are extracted within the feature extraction part. Face Detection may be challenging because of pictures and video frames will contain advanced background, completely different head poses and occlusion like carrying glasses or scarf. It presents a rule for finding face recognition downside and concatenated into one feature vector that is employed to coach the system to recognise among the prevailing photos with it. Within the testing stage the system takes the face of the image of someone for recognition. Image acquisition, preprocessing, image filtering, feature extraction is just like the learning stage. For classification the options are fed to the trained system. The algorithms can determine the face image from the content and acknowledges it.
\end{abstract}

Keywords: feature extraction, occlusion, pre-processing, image filtering.

\section{INTRODUCTION}

Nowadays advancement of man-made brainpower is effectively creating; they open up tremendous potential outcomes before us. Investigation, gauging, detection went to another level with the utilization of man-made reasoning advancements. As of late, an incredibly encouraging field of research is Computer vision. Face detection is a phase where identifying the faces from the images or video sources.

Manuscript published on January 30, 2020.

* Correspondence Author

J. Manikandan*, Assistant Professor, department of CSE, Koneru Lakshmaiah University

S. Lakshmi Prathyusha, B. Tech, CSE Department, Koneru Lakshmaiah University

P. Sai Kumar, B. Tech, CSE Department, Koneru Lakshmaiah University

Y. Jaya Chandra, B. Tech, CSE Department, Koneru Lakshmaiah University

M. Umaditya Hanuman, B. Tech, CSE Department, Koneru Lakshmaiah University.

(C) The Authors. Published by Blue Eyes Intelligence Engineering and Sciences Publication (BEIESP). This is an open access article under the CC-BY-NC-ND license (http://creativecommons.org/licenses/by-nc$\underline{\mathrm{nd} / 4.0 /)}$
It very well may be utilized for remote distinguishing proof administrations for security in regions, for example, banking, transportation, law requirement, and electrical businesses.

Despite huge varieties in visual upgrades because of evolving condition, maturing, and interruptions like whiskers, glasses, and haircut changes, this capacity is extremely powerful. In this paper we proposed a numerical model and computational model of face acknowledgment which is quick, sensibly basic, and precise in compelled condition.

Face detection utilizing Eigen face has been demonstrated to be precise and quick. With the guide of a normal web camera, a machine can identify and perceive an

individual's face; a custom login screen with the capacity to channel client get to dependent on the clients' facial highlights will be created. The goals of this is to give a lot of location calculations that can be later bundled in an effectively compact structure among the distinctive processor designs we find in machines today. This will examine PC vision, to be specific face location based Open CV library.

\section{LITERATURE SURVEY}

In the present, advances of PC vision are effectively creating, with their assistance, we can tackle issues all the more adequately, one of which is acknowledgment. Because of dynamic improvement, designers get countless libraries to take care of issues related with PC vision. Works center around the hypothetical parts of building a steady framework for face acknowledgment. This paper portrays the genuine strategies and advances for all phases of the improvement of the acknowledgment framework, since in the field of acknowledgment, an enormous number of one of a kind arrangements have been created. This paper is a utilization of face identification and following in recordings and cameras utilizing Open CV. It utilizes calculations like Adaboost, Haar course. Face identification is the most unmistakable area of research in the vision of PC building. It is a PC development which is being used in a grouping of employments that recognizes human faces in cutting edge pictures. Face identification is one of the most discussed in innovation. Face restriction can be alluded to as extraction of facial highlights utilizing design acknowledgment framework. Both MATLAB and Open CV can be utilized for execution. For the most part this paper utilizes three calculations named Haar Cascades, Cam Shift Algorithm, finding by means of movement. Out of these Haar Cascade is the best calculation in light of the fact. Initially, it was tedious, yet after the changed variant it isn't that tedious. It is effective in execution and utilized prevalently. 
Haar Cascades uses the image subtraction morphological technique to perceive the face. In this the falls of different photos of a comparative individual is taken and recorded in the database. All of the pixels in the effect of white territory are subtracted from all of the pixels in the effect of dim district. This procedure for subtraction is performed on all of the image in the course anyway all of the photos most likely won't give us the best results. This paper looks at all the calculation as far as existence worldview. It distinguishes Haar falls as the most proficient method for face discovery. As we discussed the different calculations we found that the calculations, for example, Haar falls, camshift calculation give a superior execution and a considerably more checked yield than location through movement however to the extent time is considered camshift calculation and identification by means of movement calculation is a superior alternative. Subsequently, Haar falls works gives better exactness in outward appearance.

\section{METHODOLOGY}

Face recognition takes a photo from a video or a digital camera as input and outputs the diagnosed photo subject matter. Facial features may additionally consist of regions inside the face, variations within the face structure, face cuts and angles which have been formatted and styled. Face extraction includes grabbing of the capabilities from camera. Face detection includes the elimination of the background and focusing on the foreground eliminating some other elements apart from the face vicinity, but the device nevertheless pertains some drawbacks because it cannot come across the head be counted which can be a gift because of overlapping of faces or mistaken recognition of faces having similar facial functions.

-Find faces - regardless of whether the errand of perceiving individuals in photos, or video acknowledgment, or whatever else.

-Face positioning - pics aren't regularly located on which an individual stand straightforwardly before the focus, often the face grows to become, we are facing the challenge of situating it as though the picture become taken legitimately.

-Defining outstanding facial capabilities - this development can be referred to as a full face acknowledgment step, it examinations the photograph and gets certainly one of a type automated estimations of the face.

-Identification of a person - we assessment a got information and the information efficiently accessible to us, if the statistics are similar, we will show the call of the character, if now not, in like way we've now not recognised at this point to us character. This will analyse in element each one of the means to manufacture a face acknowledgment framework and comparison their execution and the help of various libraries, simply as the velocity of crafted by way of every section in various libraries of Computer vision.

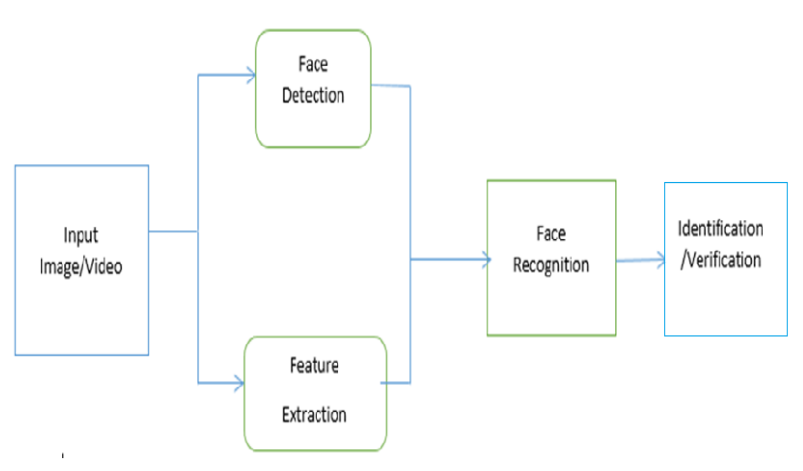

Figure 1: Block diagram of Face Detection

\section{EXPERIMENTAL INVESTIGATIONS}

The goal of our face recognition frameworks may rely upon the use of the framework. We can recognize in any event two general classes of face acknowledgment frameworks.

-We need to discover an individual inside an enormous database of countenances. These frameworks normally return a rundown of the in all likelihood individuals in the database. Regularly just one picture is accessible per individual. It is generally a bit much for acknowledgment to be done in real time.

- We need to recognize specific individuals continuously (for example in a security checking framework, banking framework, and so on.), or we need to enable access to a gathering of individuals and deny access to all others. Numerous pictures per individual are regularly accessible for preparing and constant acknowledgment is required.

In this paper, we are basically intrigued by the subsequent case. We are keen on acknowledgment with fluctuating facial detail, demeanour, present, and so on. We don't think about invariance to high degrees of turn or scaling - we accept that an insignificant pre-handling stage is accessible whenever required.

\section{IMPLEMENTATION}

A general face recognition framework incorporates numerous means:

Face Detection

Feature extraction and

Face recognition.

Face detection and recognition incorporates numerous correlative parts where each part is a supplement to the next. Contingent upon standard framework where each part can work separately. Face identification is a PC innovation that depends on learning calculations to designate human faces in advanced pictures. Face detection takes pictures/video successions as information and finds face zones inside these pictures. This is finished by isolating face regions from nonface foundation districts. 
Facial extraction finds significant highlight (eyes, mouth, nose and eye-temples) positions inside a distinguished face. A fundamental issue in face recognizable proof is the enormous contrasts between face pictures from a similar individual when contrasted with those from various people. In this manner, it is critical to pick an appropriate face order procedure that can give a decent discrete capacity between various people. Face ID has a wide scope of utilizations. Since it offers a non-meddling route for human recognizable proof, the face is utilized as a significant biometric in security applications. Open CV-Python was begun at Intel in the year 1999 by Gary Bradsky. The principal discharge came somewhat later in the year 2000. Open CV basically represents Open Source Computer Vision Library.

Despite the fact that it is written in upgraded $\mathrm{C} / \mathrm{C}++$, it has interfaces for Python and Java alongside $\mathrm{C}++$. Open CV brags of a functioning client base everywhere throughout the world with its utilization expanding step by step because of the flood in PC vision applications. Open CV-Python is the python API for Open CV. You can consider it a python wrapper around the $\mathrm{C}++$ usage of Open $\mathrm{CV}$.

Open CV-Python isn't just quick but on the other hand is anything but difficult to code and convey. This settles on it an extraordinary decision to perform computationally escalated projects.

A general explanation of the face recognition issue in Computer vision can be planned as pursues: given still or video pictures of a scene, recognize or check at least one people in the scene utilizing a put away database of appearances. Facial recognition by and large includes two phases: Face Detection where a photograph is looked to discover a face, at that point the picture is prepared to harvest and concentrate the individual's face for simpler recognition. Face Recognition where that recognized and handled face is contrasted with a database of known countenances, to choose who that individual. Since 2002, face recognition can be performed reasonably effectively and dependably with Intel's open source structure called Open CV. Face Detector that works in about $90-95 \%$ of clear photographs of an individual looking forward at the camera. Face detection anyway is substantially less solid than face recognition, with a precision of 30 to $70 \%$ as a rule. Face detection has been a solid field of research since the 1990s, yet is as yet a far route away from a dependable strategy for client verification. An ever increasing number of systems are being built up every year.

\section{DETECTOR CLASSIFIERS IN FACE}

A Computer program that chooses whether a picture is a positive picture (face picture) or negative picture (non-face picture) is known as a classifier. A classifier is prepared on a huge number of face and non-face pictures to figure out how to order another picture effectively. OpenCV furnishes us with two pre-prepared and fit to be utilized for face identification classifiers:

- Haar Classifier

- LBP Classifier
Both of these classifiers procedure pictures in dark scales, fundamentally on the grounds that we needn't bother with shading data to choose if an image has a face or not (we'll talk progressively about this later on). As these are preprepared in Open $\mathrm{CV}$, their scholarly information documents additionally come packaged with Open $\mathrm{CV} /$ information/. To run a classifier, we have to stack the information documents first, as though it had no information, much the same as a recently conceived infant (idiotic children). Each record begins with the name of the classifier it has a place with.

\section{A. HAAR CASCADES CLASSIFIER:}

The Haar Classifier is an AI based methodology, a calculation made by Paul Viola and Michael Jones; which are prepared from numerous positive pictures (with appearances) and negatives pictures (without faces).

1. Haar Feature Selection: First step is to gather the Haar Features. A Haar include considers neighbouring rectangular districts at a particular area in an identification window, summarizes the pixel powers in each area and figures the contrast between these aggregates.

2. Making Integral Images: Integral Images are utilized to make this procedure quick. A large portion of the determined highlights are superfluous.

3. Adaboost Training: An idea called Adaboost which both chooses the best highlights and prepares the classifiers is utilized. This calculation builds a solid classifier utilizing a straight blend of weighted straightforward frail classifiers.

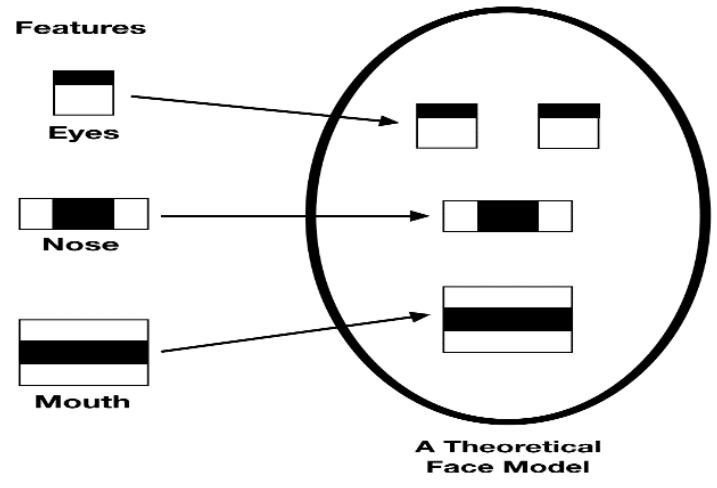

Figure 2: Detection of Theoretical Face model using Haar like features

\section{B. LBP CASCADE CLASSIFIER:}

As some other classifier, the Local Binary Patterns, or LBP to put it plainly, additionally should be prepared on many pictures. LBP is a visual/surface descriptor, and our countenances are likewise made out of miniaturized scale visual examples. Along these lines, LBP highlights are removed to frame an element vector that characterizes a face from a non-face. Each preparation picture is separated into certain squares. For each square, LBP takes a gander at 9 pixels ( $3 \times 3$ window) at once, and with a specific enthusiasm for the pixel situated in the focal point of the window. At that point, it contrasts the focal pixel esteem and each neighbour's pixel esteem under the $3 \times 3$ window. For each neighbour pixel that is more noteworthy than or equivalent to the middle pixel, it sets its incentive to 1 , and for the others, it sets them to 0 . 
From that point onward, it peruses the refreshed pixel esteems (which can be either 0 or 1 ) in a clockwise request and structures a twofold number. Next, it changes over the twofold number into a decimal number, and that decimal number is the new estimation of the middle pixel. We do this for each pixel in a square.

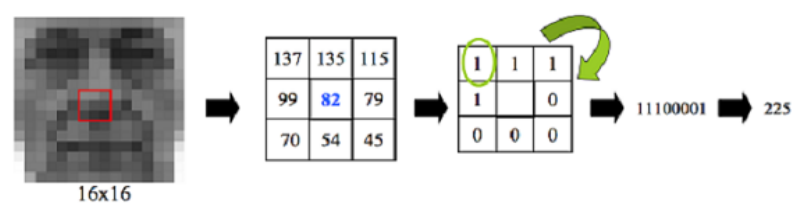

Figure 3: LBP Conversion to Binary

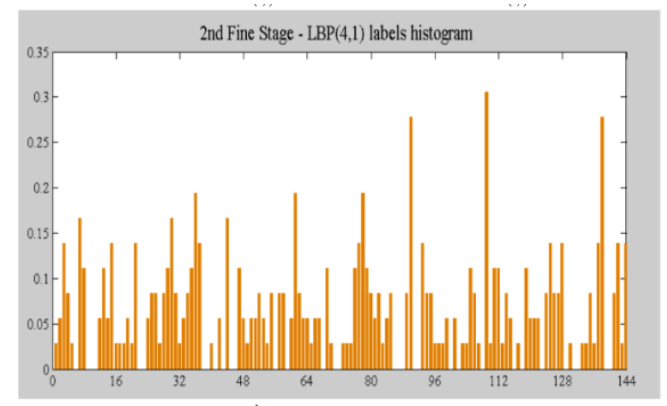

Figure 4: Histogram representation of LBP

At that point it changes over each square into histogram, so we have gotten one histogram. Finally, it connects these square histograms to shape a one element vector for one picture, which contains every one of the highlights we are intrigued. Along these lines, this is the way we extricate LBP highlights from an image.

\section{HAAR CLASSIFIER VS. LBP CLASSIFIER:}

\begin{tabular}{|c|c|c|}
\hline Algorithm & Advantages & Disadvantages \\
\hline Haar & $\begin{array}{l}\text { 1. High detection } \\
\text { accuracy } \\
\text { 2. Low false positive rate }\end{array}$ & $\begin{array}{l}\text { 1. Computationally complex } \\
\text { and slow } \\
\text { 2. Longer training time } \\
\text { 3. Less accurate on black } \\
\text { faces } \\
\text { 4. Limitations in difficult } \\
\text { lightening conditions } \\
\text { 5. Less robust to occlusion }\end{array}$ \\
\hline LBP & $\begin{array}{l}\text { 1. Computationally simple } \\
\text { and fast } \\
\text { 2. Shorter training time } \\
\text { 3. Robust to local } \\
\text { illumination changes } \\
\text { 4. Robust to occlusion }\end{array}$ & $\begin{array}{l}\text { 1. Less accurate } \\
\text { 2. High false positive rate }\end{array}$ \\
\hline
\end{tabular}

\section{FACE-RECOGNITION ALGORITHMS}

\section{A. EIGEN FACES ALGORITHM:}

Since we have a pre-processed facial picture, we can perform Eigenfaces (PCA) for Face Recognition. OpenCV accompanies the capacity "cvEigenDecomposite()", which plays out the PCA activity, anyway we need a database (preparing set) of pictures for it to realize how to perceive every one of your kin. Use "Head Component Analysis" to change over the preparation pictures into a lot of "Eigenfaces" that speak to the fundamental contrasts between the preparation pictures. First it will locate the "normal face picture" of your pictures by getting the mean estimation of every pixel. At that point the eigenfaces are determined in contrast with this normal face, where the first eigenface is the most prevailing face contrasts, and the second eigenface is the second most predominant face contrasts, etc., until you have around 50 eigenfaces that speak to the greater part of the distinctions in all the preparation set pictures.

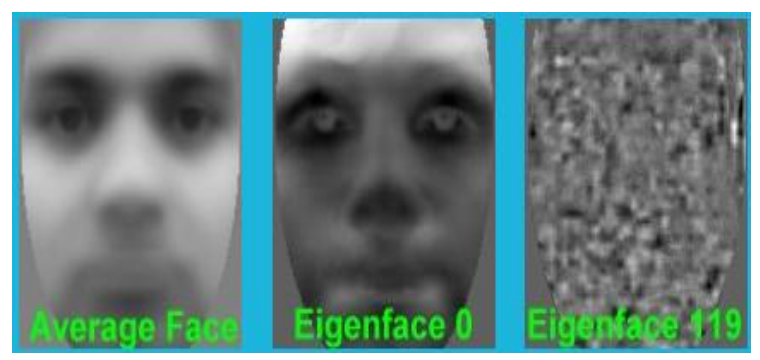

Figure 5: Eigen Face representation for Face Recognition

In these model pictures above you can see the normal face and the first and last eigenfaces that were produced from an assortment of 30 pictures every one of 4 individuals. Notice that the normal face will show the smooth face structure of a nonexclusive individual, the initial not many eigenfaces will give some prevailing aspects of countenances, and the last eigenfaces (e.g.: Eigenface 119) are for the most part picture clamour. You can see the initial 32 eigenfaces in the picture underneath.

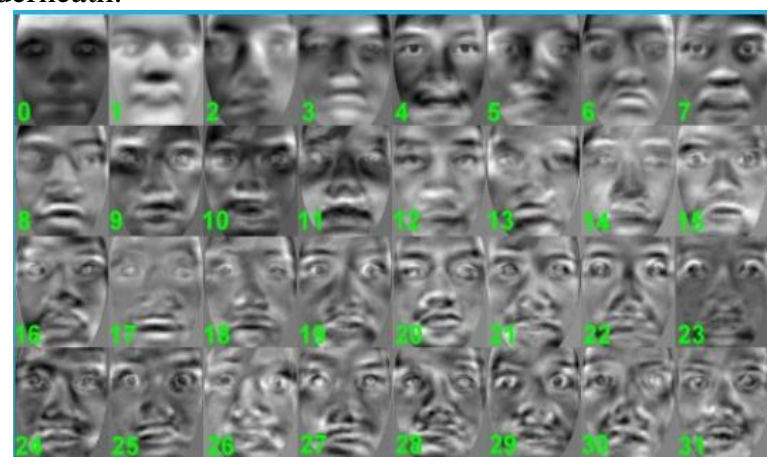

Figure 6: Eigen representation for initial 32 faces

\section{B. FISHERFACES ALGORITHM:}

The Principal Component Analysis (PCA), which is the center of the Eigenfaces technique, finds a direct blend of highlights that expands the all-out difference in information. While this is plainly an incredible method to speak to information, it doesn't think about any classes thus a great deal of discriminative data might be lost when discarding segments. The Fisherfaces strategy learns a class-explicit change grid, so the they don't catch brightening as clearly as the Eigenfaces technique. The Discriminant Analysis rather finds the facial highlights to separate between the people. It's critical to make reference to, that the exhibition of the Fisherfaces intensely relies upon the info information too. For all intents and purposes stated: on the off chance that you become familiar with the Fisherfaces for wellenlightened pictures just and you attempt to perceive faces in terrible lit up scenes, at that point technique is probably going to locate an inappropriate part (in light of the fact that those highlights may not be overwhelming on awful lit up pictures). This is to some degree consistent, since the technique got no opportunity to get familiar with the brightening. 


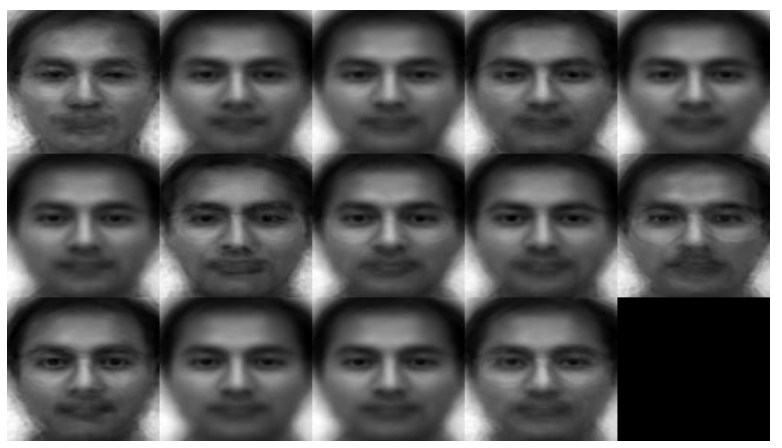

Figure 7: Fisher faces representation for face recognition

\section{RESULTS}

The last form of the trial is a face recognition framework which depends on OpenCV. The consequences of their work is given underneath which gives an interface of utilizations, wherein we see the detected face.

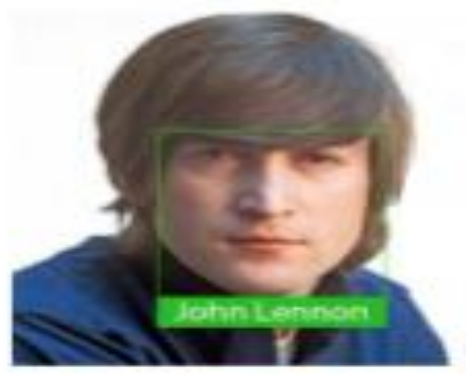

Figure 8: Example of Face Detection

In light of the investigation of the submitted OpenCV, it has been set up that there is no single strategies and advancements to make a dispersed acknowledgment data framework that would join all phases of the framework development. There is an enormous number of techniques for looking, situating and as a rule for sorting out the acknowledgment procedure. By picking innovations you ought to be exceptionally cautious in light of the fact that relying upon your needs you should utilize certain strategies. Thusly, work with a nitty gritty depiction of advancements for acknowledgment is very important at present, just as the improvement of new advances and an approach to take care of this squeezing issue. As referenced above, presently the innovation of PC vision and devices for face recognition are effectively creating. Worldwide innovation pioneers put resources into innovative work a great deal of cash since this issue has wide open doors for use. The course for the improvement of face acknowledgment is principally the security and expanded assurance frameworks. At present the most utilized in amusement, I accept this pattern will develop too. Proposed approach can be utilized for enormous information handling.

\section{CONCLUSION}

According as far as our knowledge is concerned, the OpenCV library is progressively profitable, has better execution for face location and recognition. It additionally implies that with OpenCV, it's smarter to assemble acknowledgment applications for the IOT stage. Note that lone HOG calculation has been investigated while scanning for different calculations, for example, the Haar course, it works longer, yet turns out additional in detail, if there are a lot of photographs later on for some, it is prudent to consider utilizing this strategy. Nonetheless, the structure rationale and the key purposes of making an application acknowledgment, were talked about. Haar Cascade Classifier works better which has the best exactness when contrasted with some other calculations like LBP and so on.,

\section{REFERENCES}

1. Face Detection and Recognition using OpenCV, Article, http://shervinemami.info/faceRecognition.html, Published by Shervin Emami

2. OpenCV: OpenCV Tutorials [Electronic resource] https://docs.opencv.org/master/d9/df8/tutorial_root.html

3. R. Raja, Face Detection Using OpenCV and Python [Electronic resource] - Access mode: Facial_Recognition_using_OpenCV (1).pdf

4. Neetu Saini, Sukhwinder Kaur, Hari Singh (2013). A Review: Face Detection Methods and Algorithms, International Journal of Engineering Research \& Technology (IJERT) ISSN: 2278-0181 https://www.ijert.org Vol. 2 Issue 6.

5. Hemant Makwana \& Taranpreet Singh," comparison of different algorithm for face recognition". "global Journal of Computer Science and technology graphics \& vision. Volume 13 Issue 9 Version 1.0 Year 2013

6. Wikipedia, Active appearance model, http://en.wikipedia.org/wiki/Active_appearance_model

7. Wikipedia, Histogram equalization, https://en.wikipedia.org/wiki/Histogram_equalization

8. Face Detection and Tracking Using OpenCV "International Conference on Electronics, Communication and Aerospace Technology ICECA 2017.

\section{AUTHORS PROFILE}

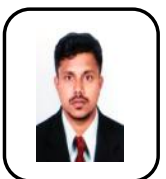

J. Manikandan is working as an Assistant Professor in department of CSE in Koneru Lakshmaiah University. His research area is Big Data and data Analytics. He is having experience in teaching Area of interest in subjects Database Systems, Design and Analysis of Algorithms, Knowledge Engineering and IOT etc.,

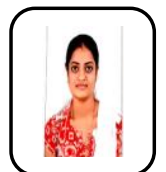

S. Lakshmi Prathyusha is pursuing final year B. Tech of CSE Department in Koneru Lakshmaiah University. Her research area is Big Data and data Analytics. She is a ServiceNow Certified Developer and participated in hackathon. Her areas of interest are OOPS, Software Engineering, Database Systems

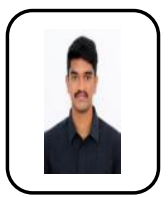

Y. Jaya Chandra is pursuing final year B. Tech of CSE Department in Koneru Lakshmaiah University. His research group is Data Analytics. He is a ServiceNow Certified Application Developer. His areas of interest are Database Systems, OOPS in Java, Analysis of Algorithms etc.,

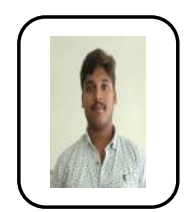

M.Umaditya Hanuman is pursuing final year B. Tech of CSE Department in Koneru Lakshmaiah University. His research area is Data Analytics. He is ServiceNow Certified Developer. His areas of interest are Artificial Intelligence, Java, Python and etc.,

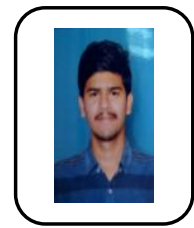

P. Sai Kumar is pursuing final year B. Tech of CSE Department in Koneru Lakshmaiah University. His research area is Big Data \& Data Analytics. His areas of interest are OOPS with Java, UI Path, Database management systems, Operating Systems and etc. 